\title{
Caractérisation du tenseur des contraintes naturelles : bases théoriques et retours d'expérience
}

\section{P. SIRIEYS ${ }^{\dagger}$}

13, rue de la République 38000 Grenoble psirieysorange.fT

\section{FABRE}

Conservatoire national des arts et métiers Chaire de Géotechnique 2 , rue Conte, CP 341 75141 Paris Cedex 3 denis.fabre@cnam.fr

M. SAHLI

EHTP. Dépantement PCT BP 8108 Casablanca

Maroc

sahligehtp.ac.ma

B. MAYEUR

Coyne et Bellier 92632 Genneviliers Cedex bertrand.mayeur@coyneet-bellier.fi
Létat de contraintes actuel, dont la modification anthropique ou naturelle entraine des déformations, éventuellement des césordres, est un des éléments essentiels de la reconnaissance d'un terrain. Sa détermination fait appel ả des bases thériques et à l'expérimentation.

Dans la premiêre partie, outre quelques rappels bibliographiques concernant les champs théoriques pour des surfaces topographiques planes ou diédriques, est introduit le tenseur ( $K_{n}$ ) qui se ramène at scalaire $k_{0}$ dans le cas du milieu pulvérulent. Le tenseur de surface $\left(\Sigma_{0}\right)_{0}$ conduil à une nouvelle définition de $k_{\mathrm{u}}$ pour les milleux cohésifs. Enfin, une étude originale en élasticité est proposée pour les vallées en $\mathrm{U}$ et $\mathrm{V}$ et les massifs en éperon ou en aiguille.

La deuxième partie traite des reliefs de type vallée. montagne ou mixte par modélisation numérique. Sont présentés des exemples avec tracé des isostatiques et, sur une isostatique verticale, les variations des contraintes principales verticale et horizontale $\left[\sigma_{v}\right.$ et $\left.\sigma_{H}\right\}$. L'effet d'un serrage latéral $\alpha$ tectonique $n$ est analysé. La troisième partie a trait à des retours d'expérience français récents ; elle confronte résultats analytiques et numérigues avec mesures de terrain. L'Est die la France est concerné par une extension qui peut se manifester en surface par une contrainte principale mineure négative (traction). Dans le cas des Alpes, les effets des reliefs et de la tectonique se font sentir : inclinaison du trièdre principal sous les versants (parallélisme entre versant et isostatique majeure) ; concentration des contraintes horizontales sous les vallées (souvent perpendiculairement à celles-ci) : pour les contraintes verticales : effets d’alourdissement sous les vallées el d'aliégement sous les montagnes.

Mots-clés : contraintes, triẻdre principal, orthotropie, majeure-mineure-intermédiaire, isostatiques, mesure des contraintes, méthodes hydrauliques, relief, conditions. tectoniques.
NDLR : Les discussions sur cet article sont acceptees jusqu'au 1'mars 2009
The actual state of stresses, of which the anthropic or natural modification drags some distortions, is one of the essential elements of the soil recognition. Its determirjation calls on theoretical bases and experimentetion.

In the first part, besides some bibliographic recalls concerning the theoretical fields under plane on cliedric topographic surfaces, the tensor $\left(\mathrm{K}^{\prime}\right)$ is introduced that reduces to the scalar $k_{\text {for }}$ granular media. The surface tensor [ $\left.\Sigma_{\text {p }}\right)_{0}$ ] leads to a new definition of $k_{\text {for }}$ cohesive media. An original elastic analytical study is also proposed for valleys in * $\mathrm{U}$ ) and a $\mathrm{V}$ \% and mássif's in a spur a or \& needle n shape. 


\begin{tabular}{|c|c|}
\hline & MOTATIONS \\
\hline$\left(v_{i s}\right)$ & : tenseur des contraintes natureiles \\
\hline$\left(\Sigma_{0}\right)_{0}$ & $\begin{array}{l}\text { : tenseur des contraintes en surface } \\
(z=0)\end{array}$ \\
\hline$(\mathrm{H}, \mathrm{h}, \mathrm{v})$ & $\begin{array}{l}\text { : trièdre principal de }\left(\Sigma_{\mathrm{v}}\right) \text {, lorsque la } \\
\text { verticale } z \text { est direction principale }\end{array}$ \\
\hline$(I, \Pi,[I I)$ & $\begin{array}{l}\text { trièdre principal de }\left(\Sigma_{p}\right) \text {, avec l] hori } \\
\text { zontal, perpendiculaire au plan } x z\end{array}$ \\
\hline$\gamma$ & : poids volunique du terrain \\
\hline c. $\phi$ & $\begin{array}{l}\text { cohésion et angle de frottement } \\
\text { pour un matériau obéissant ả la loi } \\
\text { de Coulomb }\end{array}$ \\
\hline$\left(\sigma_{\mathrm{H}^{\prime}}, \sigma_{h}, \sigma_{\mathrm{\gamma}}\right)$ & $\begin{array}{l}\text { Contraintes principales, Jorsque z } \\
\text { est direction principale }\end{array}$ \\
\hline$\left(\sigma_{\mathrm{HO},}, \sigma_{1,0}\right)$ & $\begin{array}{l}\text { : valeurs des contraintes horizontales } \\
\text { en sufface }\end{array}$ \\
\hline$k_{1 j}$ & $\begin{array}{l}\text { rapport des contraintes effectives } \\
\sigma_{1}^{\prime} / \sigma_{y}^{\prime}\end{array}$ \\
\hline$k_{0 x}$ & $\begin{array}{l}\text { rapport des contraintes pour un sol } \\
\text { élastique lorsque } \varepsilon_{x}=0\end{array}$ \\
\hline$k_{\text {OH }}$ & $\begin{array}{l}\text { : gradient du rapport des contraintes } \\
\text { dans la direction } \mathrm{H}\end{array}$ \\
\hline$k_{0 \text { on }}$ & $\begin{array}{l}\text { : gradient du rapport des contraintes } \\
\text { dans la direction h }\end{array}$ \\
\hline$\left[\mathbf{K}_{0}\right]$ & : tenseur adimensionnel \\
\hline
\end{tabular}

NB : selon l'usage en géotechnique, les compressions sont positives et les rractions négatives.

\section{1}

\section{Introduction}

Le terrain, dans sa configuration actuelle, est le sìge d'un état de contraintes, dit naturel et noté $\left(\Sigma_{0}\right)$. Parfois, notamment dans les formations superficielles sous certaines conditions (géométriques et mécaniques), ( $\Sigma_{\emptyset}$ ) peut être défini à l'aide d'un scalaire noté $k_{\text {u. }}$ Mals, dans les massifs de roches indurées, son
The second part is about realistic reliefs ivallev. mountain or combined) using numerical modeling. Some examples are presented showing the isostatic lines and, on a vertical isostatic, the variations of the main vertical and horizontal stresses $[\sigma$, and $\sigma_{,}, 1$. The effect of a istonic $n$ lateral tightening is analyzed. The third part deals with recent returns of French expertience : it confronts analvtical and numerical results with field measurements. The East of France is concerned with a regional extension that may even appear in surface by a main minor negative stress (traction]. In the case of the Alps, the topographic and tectonjc effects are sensitive : il slant of the principal reference axes under the sides (parallelism betwern relief side and majol isostatic) : ii) concentration of the horizontal stresses under the valleys (often perpendicularly to these) : iii\} for the wertical stresses : a heaviress effect under the valieys and o lightness effect undar the hourtatins.

Key words : stresses, principal direction axes, orthotropy. major-minor-intermediate, isostatic lines, stress measurements, hydraulic methods, mountainous relief intluence. tectonic conditions. expression doit être adaptée à la réalité géologique. Les résultats de l'analyse des mesures au vérin plat montrajent déjả cette complexité en surface (Hoek et Brown, 1980, Froidevaux et al., 1980). Plusieurs publications récentes en France soulignent l'importance d'une meilleure connaissance du champ de contraintes naturel pour le calcul des ouvrages géotechniques (CFMR, 2000, Sirieys, 2001. Fabre et al., 2002 ; AFTES, 20031. Un numéro spécial de la revue Intemational Journal of Rock Mechanics \& Mining Sciences (2003, $n^{-7} 7-8$ ) est consacré aux contraintes avec une synthẻse historique (Fairhurst, 2003), de nombreux exemples de résultats et la présentation des méthodes de mesure recommandés (a ISRM suggested methods for rock stress estimation $)\}$.

Cet article souligne cependant les éléments theoriques qui permetten de caractériser complètement le tenseur de contraintes nahurelles dans les divers types de terrains (sols et roches) et pour des géométries simples de la sưface. UIn essai de modélisation numériçue des effets de la topographie et des conditions tectoniques est présenté. Des résultats de récentes campagnes de mesures dans l'Est et le Sud-Est de la France illustrent entin la variabilité des champs naturels.

\section{2}

\section{Résultats théoriques: solutions analytiques}

Dans les massifs rocheux en général, incluant les roches meubles $\left(\Sigma_{\gamma}\right)$ est caractérisé par

- le comportement mécanique du terrain : élasticité: atteinte de l'équilibre limite ; anisotropie (induite notamment par lia déformation tectonique) :

- les conditions aux limites : géométrie de la surface topographique, chargements éventuels..

Les champs peuvent être explicités par des solu tions analytiques, seulement pour des surfaces à géométrie simple (plans, dièdres et rectangles en relief ou en creux). 


\section{Massif semi-infini à surface libre horizontale}

\author{
Milieu pulvérulent, homogène, isotrope, \\ en régine élastique
}

\section{a) Le scalaire $\mathrm{k}_{\mathrm{y}}$}

Dans le cas des sols meubles, $\left(\Sigma_{\mathrm{y}}\right)$ est considéré comme de révolution autour de la direction principale verticale z (isotropie dans le plan horizontal). Dans le référentiel $(x, y, z)$, en un point de cote $z$ [profondeur), les équations de l'équilibre conduisent à l'expression de la contrainte likhostatique verticale:

$$
\sigma_{z}=\gamma z
$$

résultat encore appelé a poids des terres h ou a poids de colverture $)$.

Le tenseur $\left(\Sigma_{p}\right)$ est défini complètenent, lorsque la contrainte principale horizontale os, est connue ; il est caractérisé par un paramètre unique : le scaiaire $k_{0^{2}}$ appelé « coeffícient de pression des terres au repos". défini рar:

$$
k_{v}=\frac{\sigma_{10}}{\sigma_{v}}
$$

ou $k_{v}=\frac{\sigma_{h}^{\prime}}{\sigma_{v}^{\prime}}$ en milieu saturé, en faisant intervenir les contraintes intergranulaires.

Les composantes principales du tenseur des contraintes sont alors $\left(k_{0} \gamma z, k_{0} \gamma z, \gamma z\right)$ et le champ des contraintes s'exprime par":

$$
\sigma_{\mathrm{g}}=\gamma \mathrm{z} \quad \sigma_{\mathrm{h}}=k_{\mathrm{g}} \gamma \mathrm{z}
$$

Lhypothèse d'une déformation horizontale nulle (condition appelée « œedométrique » en mécanique des sols, et « lithostatique " en géologie), en milieu élastique (v étant le coefficient de Polsson), condujt à une valeur particulière notée $\mathrm{k}_{02}$ :

$$
k_{0 x}=\frac{v}{1-v}
$$

soit pour $\mathrm{v}=1 / 3, \mathrm{k}_{\mathrm{ou}}=0.5$.

$k_{\text {v }}$ varie dans le temps (une contraction horizontale l'accroit, une extension le faìt décroitre) dans des limites définies par le critère de linute élastique, soit. pour un sol répondant au critère de Coulomb (angle de frottement $\varphi$ ):

avec $k_{\mathrm{a}}=\frac{1-\sin \varphi}{1+\sin \varphi} \quad \mathrm{k}_{\mathrm{p}}=\frac{1+\sin \varphi}{1-\sin \varphi}$

$\mathrm{k}_{0}$ est donc encadré par deux valeurs linites (pour $\varphi=30^{\circ}: 1 / 3<k_{0}<3$ ).

\section{b) Le tenseur $\left(K_{0}\right)$}

En milieu pulvérulent, les conditions aux limites de surface (pour $z=0, \sigma_{\mathrm{p}}=\sigma_{\mathrm{h}}=0$ ) permettent de normer $\left(\Sigma_{0}\right)$ par $\gamma z$, soit :

$$
\left(\Sigma_{0}\right)=\gamma z\left(K_{0}\right)
$$

La relation (3) définit un tenseur $\left(K_{r}\right)$ de révolution autour de la verticale, de composantes principales adimensionnelles $\left(\mathrm{k}_{0,}, \mathrm{k}_{\mathrm{QY}}, \mathrm{i}\right)$.

L’isotropie des contraintes horizontales n'est que rarement vérffiée, notamment par suite d'un épisode tectorique : serrage ou extension dans une direction. L'état orthotrope se caractérise par son triedre principal $(v, H, h)$, c'est-ä-dire par la verticale v $(=z)$ et dewx directions orthogonales majeure (H) et mineure (h) dans le plan horizontal ( $x$ y). dont l'orientation ne dépend que d'un paramètre angulaire $\alpha=(x, H)$ (Fig. 1).

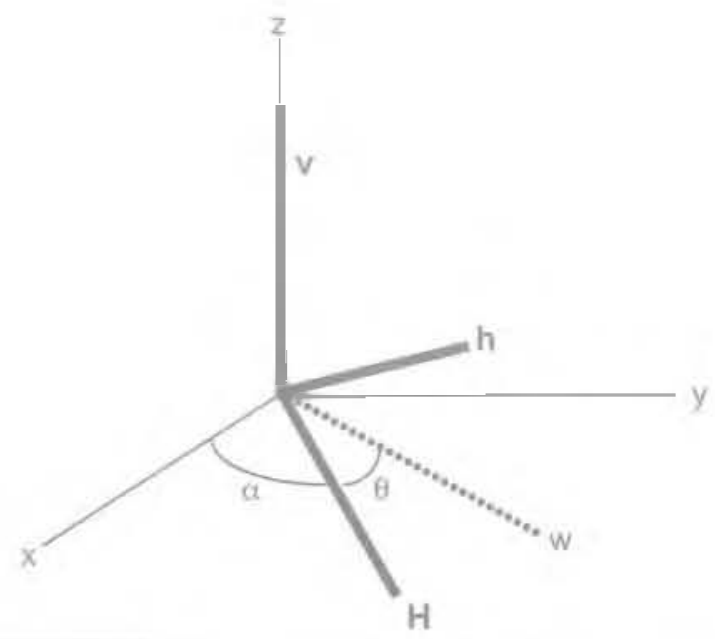

FG. 1 Cas de l'orthotropie. notations : triedre principal $(H, h$. v) el direction quelconque w dans le plan horizontal.

Notations (orthotropy] : principal trihedron l. H. h, w) : any direction i wi in the horizontal plathe.

Les composantes principales de $\left[\Sigma_{0}\right)$ sont : $\sigma_{z}\left(=\sigma_{v}\right)$ $\sigma_{H}$ et $\sigma_{h}\left(\right.$ avec $\left.\sigma_{H}>\sigma_{\mu}\right)$.

Une variation linéaire avec 2 de ces trois composantes conduil aux trois gradients principaux : $\gamma, \gamma \mathrm{k}_{\text {0H }}$ $\gamma k_{\text {[nin }}$

$\left(K_{n}\right)$ est alor's caractérisé par ses trois composantes

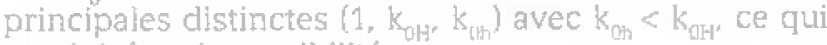
conduit à troís possibilités :

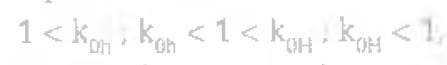

lesquelles correspondent respectivement à trois cas de la direction primcipale z lz mineure : z intermédiaire; z maleurel.

Dars une direction horizontale quelconque $\mathrm{W}_{\text {, repé- }}$ rée par l'angle $\theta=(H, W)$ (Fig. 1$\}$, la contrante horizontale $\sigma_{\theta} s^{2}$ exprime par:

$\sigma_{\theta}=\left(k_{O H} \cos ^{2} \theta+k_{0 \mathrm{~L}} \sin ^{2} \theta\right) y z=k_{0 \Theta} Y z$

Le coefficient $k_{\text {man }}$ dêfini par (4), est intermediaire entre $k_{\text {Lin }}$ et $k_{\text {oH }}$. Les valeurs moyenne $k_{0 m}=\frac{k_{0 H}+k_{0 h}}{2}$ et déviatoire $k_{01}=\frac{k_{0 H}-k_{0 h}}{2}$ caracterisent l'intensité et Janisotropie du champ de contraintes.

La variation des contraintes en fonction de la prom fondeur est représentée figure 2a pour le cas $\mathrm{K}_{U H}<1$. 


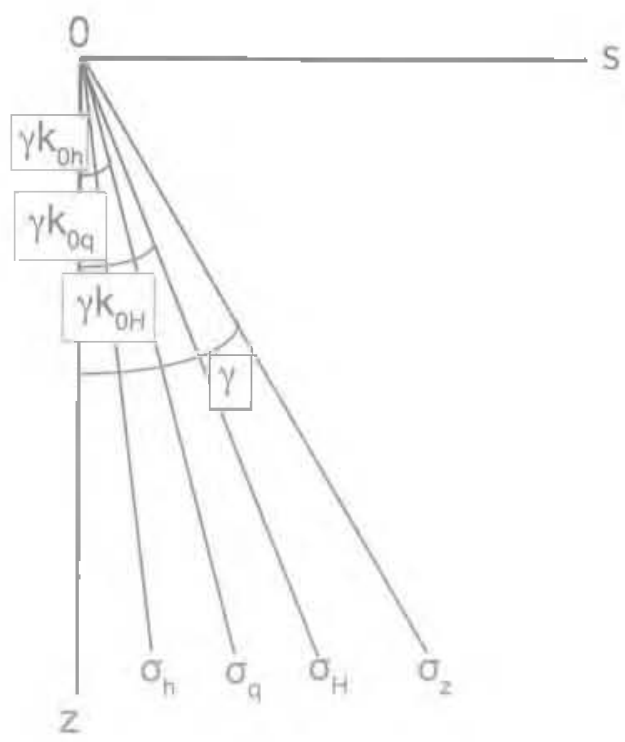

(a)

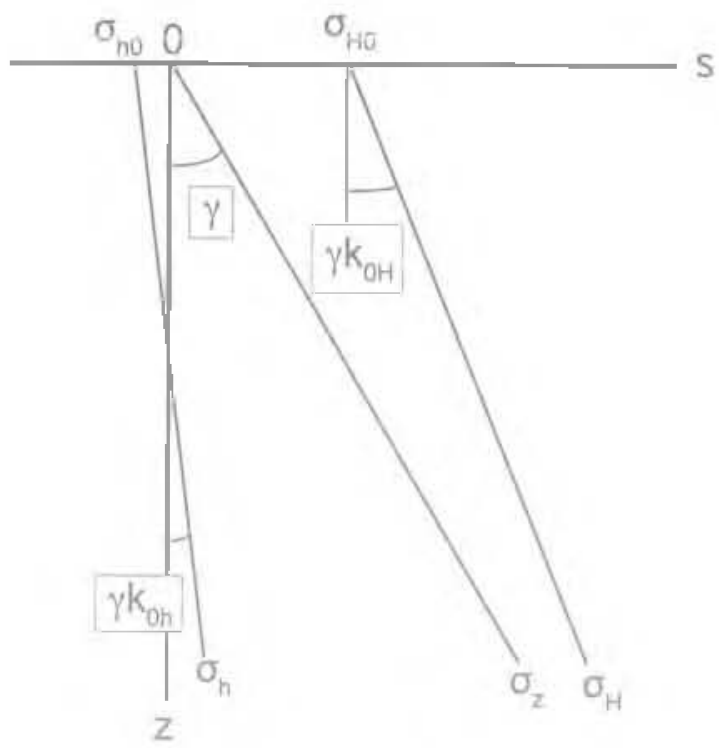

(b)

FG. 2 Variations des composantes de $\left(\Sigma_{0}\right)$ avec la profondeur dans le cas de l'orthotropie. a) Milieu puivérulent ; b) Milieu coherent (cas $a_{h 0}<0$ ). Variation of ( $\Sigma$ ) components is depth (orthotropy). a) Non cohesive material ; b) Cohesive material (with $\sigma_{h o}<0$ )

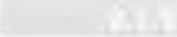

\section{Milieu cohérent (sols indurés et roches compactes)}

a) Isotropie du tenseur ( $\left.\Sigma_{0}\right)$ dans le plan horizontal

En surface $\sigma_{,}=0, \sigma_{n 0}$ est non nulle, en général une faible compression. Le champ s"écrit alors

$$
\sigma_{z}=\gamma z \quad \sigma_{I I}=\sigma_{h}=k_{0} \gamma z+\sigma_{h 0}
$$

La définition de $k_{0}$ par la relation (1), qui s'écrirait :

$$
\frac{\sigma_{h}}{\sigma_{i}}=k_{0}+\frac{\sigma_{h 0}}{\gamma z}
$$

et conduirait à faible profondeur à des valeurs infinies (tendance remarquée d'après les mesures par Hoek et Brown 1980...) r'est pas acceptable. En considérant une variation linéaire de $\sigma_{4}$ et $\sigma_{v}$ avec $z$, la définition (1) doit être remplacée par

$$
\mathrm{k}_{\mathrm{j}}=\frac{\sigma_{11}-\sigma_{\mathrm{k0}}}{\sigma_{4}}
$$

$\sigma_{\text {mo }}$ (nulle pour un sol pulvérulent) est compris entre 2 valeurs qui résultent de l'atteinte de l'état línte en plasticité, soit pour un matériat de Coulomb, avec les coefficients $k_{\mathrm{a}}$ et $k_{\mathrm{i}}$ définis ci-dessus :

$$
-2 c k_{\mu} \frac{1}{2}<\sigma_{n 0}<2 c k_{p} \frac{1}{2}
$$

soit, pour $\varphi=30^{\circ}=-1,15 c<0_{h 0 l}<3,46 c$

Généralement, $\sigma_{\mathrm{n}}$ est positive : de rares structures circulaires en systeme extensif pourraient correspondre à une traction. tal

b) Orthotropie du tenseur $\left[\Sigma_{\mathrm{p}}\right\}$ dans le plan horizon-

Avec la condition de surface, le champ s'écrit

$$
\left(\Sigma_{0}\right)=y z\left(K_{0}\right)+\left(\Sigma_{0}\right\}_{0}
$$

$(\Sigma)_{u}$, état de contraintes en surface, a pour trièdre princi. pal $(\mathrm{H}, \mathrm{h}, \mathrm{z})$ et pour composantes principales $\left(\sigma_{\mathrm{H}, \mathrm{y}} \sigma_{\mathrm{ht1}} 0\right)$.

Comme dans le cas des sols pulvérulents en champ orthotrope. $\left(\mathbf{K}_{0}\right)$ a comme composantes principales $\left(k_{v a}, k_{0,}, 1\right]$. La composante principale mineure de $[\Sigma]_{4}$ peut être une traction $\left(\sigma_{n}<0\right)$, comme le montrent certains retours d'expérience (\$4). L'exemple théorique de la figure $2 \mathrm{~b}$ correspond à cette situation

En état d'équillibre limite, trois cas se présentent. selon les valeurs relatives de $k_{0,3}$ et $k_{0 H}$ par rappork à 1. les structures géologiques relatives à ces trois cas (Fig. 3) étant les failles normales (si conjuguées: « graben $m$, les failles inverses ( chevauchements $x$ ) et les décrochements.

Concernant les orientations principales:

$-\mathrm{k}_{y+}<1$ [ Fig. 3a) : H est la direction principale intermédiaire, son azimut est celui des failes normales ; il y a eu extension dans la direction $h$ :

$-1<k_{\text {on }}$ (Fig . 3b) : h est l'intermédiaire ; Je milleu a subi une contraction dans la direction $\mathrm{H}$; deux directions de failles inverses peuvent exister :

$-\mathrm{k}_{0 \mathrm{~b}}<1<\mathrm{k}_{\mathrm{g} w}$ (Fig. 3c) : z est l'intermédiaire; il $\mathrm{y}$ a deux familles de décrochements conjugués (dextres et senestres) verticaux.

Dans le cas d'une contraction horizontale, l'atteinte cle la limite élastique peut done se manifester de deux façons : décrochement ou chevauchement, selon la direction de ta contrainte intermédiaire.

\section{9}

\section{Milieu semi infini à surface libre d'inclinaison i sur l'horizontale (rappel de quelques solutions analytiques)}

En déformation plane, dans le référentiel (n, t) (Fig. 4.1a), Je tenseur des contraintes noté ( $\Sigma)_{\text {in }}$ qui s'exprine par : $(\Sigma)_{n t}=\left(\begin{array}{ll}\sigma_{n} & \tau_{n t} \\ \tau_{n t} & \sigma_{t}\end{array}\right)$ verifie les équations d'équilibre statique d'un milieu pesant : 

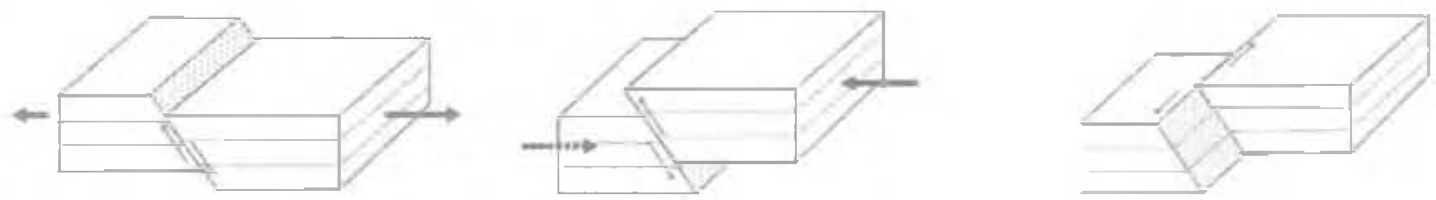

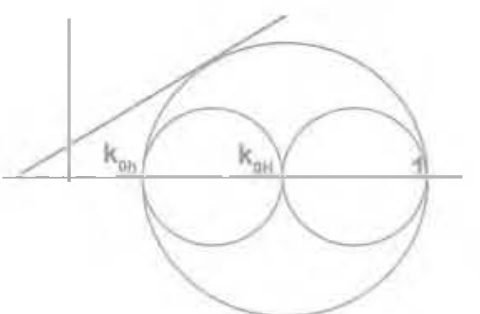

(a)

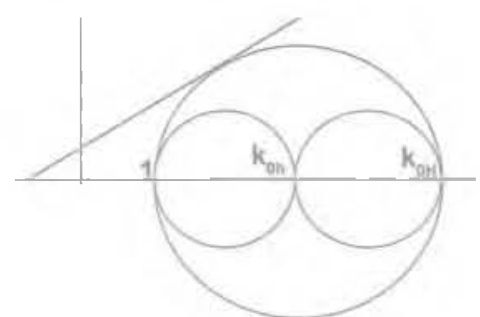

(b)

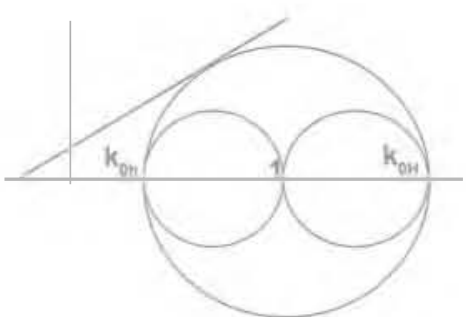

(c)

F19. 8 Structures géologiques et tenseurs limite $\left(\mathrm{K}_{p}\right)$ associés.

a) Faille normaie; b) Faille inverse :

c) Décrochement (senestre).

Geological structures with associated linit tensors.

a) Normal fautt ; b. Reverse fault ; c) Strike-5lip fault.

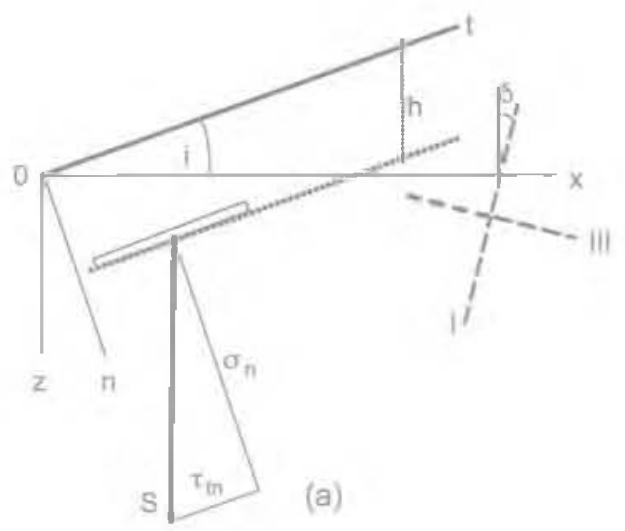

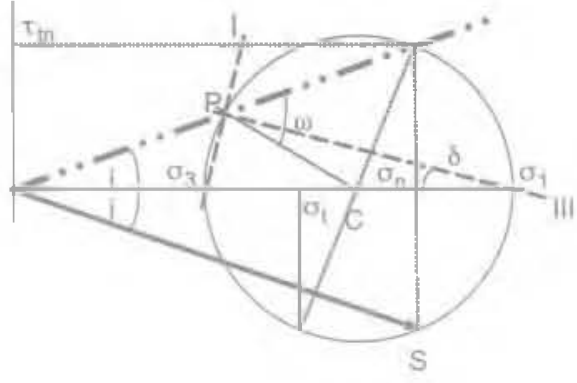

(b)

Fig. 4.1

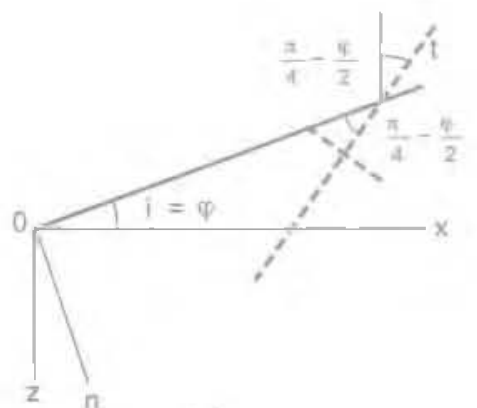

(a)

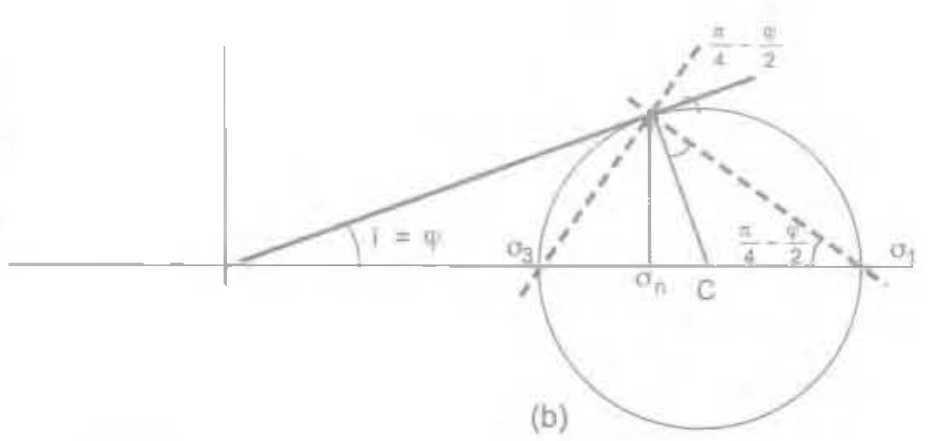

Fig. 4.2

FG. 4 Contraintes sous un milieu semi infini d'inclinaison i.

4.1. Elastique $\{0<\mathrm{I}<\mathrm{p}\}$

4.2. Plastique $[(\mathrm{c} \equiv 0$, cas $\mathrm{i}=\mathrm{q})$.

a) directions des contraintes principales ; b] construction de Mohr dans le cas ot̉ $\sigma_{n}>\sigma_{1}$ "

Stresses for semi-finfinite medium under inclined surface (argle il.

4.1. Elastic conditions $[0<1<$ Q $]$.

4.2. Plastic conditions (case $l=\$ ; c=0$ )

a) directions of principal stresses : bl Mohr circle for $\sigma_{n}>\sigma_{\text {a }}$ 


$$
\begin{aligned}
& \frac{\delta \sigma_{n}}{\delta n}+\frac{\delta \tau_{i n}}{\delta t}=\gamma \cos i \\
& \frac{\delta \tau_{n t}}{\delta n}+\frac{\delta \sigma_{t}}{\delta t}=-\gamma \sin i
\end{aligned}
$$

Avec l'hypothèse selon laquelle $(\Sigma)$ est indépendant de t, et Ot étant une surface libre:

$$
\begin{aligned}
& a_{n}=\gamma h \cos ^{2} i \\
& \tau_{n}=-y h \cos i \sin i
\end{aligned}
$$

Selon la loi de comportement, les champs de contraintes ont des caractéristiques (orientations. valeurs) différentes:

a) En élasticité de Hooke et en condition u oedonétrique $n$, les contraintes principales s'écrivent analytiquement en fonction de $\sigma_{n}$, du scalaire $k_{0 \alpha}\left(k_{a b}=\frac{v}{1-v}\right)$

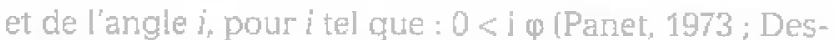
cceudres, 1984). La direction principale majeure fait avec la verticale un angle $\delta$ dont l'expression analytique est (Fig. $4.1 \mathrm{a}$ et $4.1 \mathrm{~b}$ ) :

$$
\delta=\frac{\omega-i}{2} \text { avec } \sin \omega=\frac{\left(1-k_{0 a}\right) \sin i}{\sqrt{\operatorname{tg}^{2} i+\frac{\left(1-k_{(a)}\right)^{2}}{4}}}
$$

b) Dans le cas d'un écoulement isovolume, l'angle 8 $\operatorname{vaut}\left(\frac{\pi}{4}-i\right)$ (Goguel, 1942)

c) Pour un sol en état limite [Coulomb) et pour i te] que $0<i<\varphi$, l'angle $\delta$ vaut $\frac{\theta-i}{2}$, avec $\sin \theta=\frac{\sin i}{\sin \varphi}$ (Sokolovski 1965),$\delta=\left(\frac{\pi}{4}-\frac{\varphi}{2}\right)$ dams le cas particulier $i=\varphi$ [Fig. 4.2).

Dans les trois cas, les champs sont rectilignes et les isostatiques, enveloppes des directions principales appelées aussi a trajectolies des contraintes principales $n$, sont des droites obligues par rapport au versant.

\section{3}

\section{Surface topographique en forme de dièdre (brève revue bibliographique)}

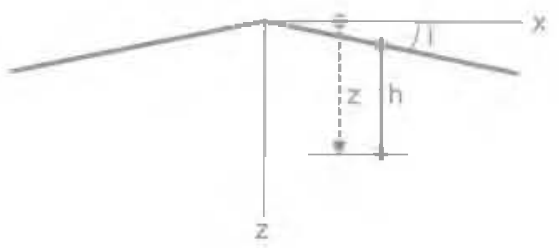

(a)

\section{Dièdre symétrique d'angle $<\pi$ à flancs inclinés à i (u montagne "Fig. 5a)}

a) Un exemple de solution élasticue obteru ả l'aide d'une fonction d'Airy polynomiale du $3^{g}$ degré (Davis et Salvadurai, 1996) est celui de Levy (in Panet, 1973), pour lequel :

$$
\begin{aligned}
& \sigma_{x}=\frac{1}{2} \gamma h \cot ^{2} \mathrm{j} \\
& \sigma_{z}=\frac{1}{2} \gamma h \\
& \tau_{x z}=\frac{1}{2} \gamma x
\end{aligned}
$$

Le champ de Lévy est curviligne et de type linéaire : $\Sigma_{4}$ croit linéairement avec les coordonnées; les faces du dièdre sont des isostatiques.

Sur Oz, direction principale, la contrainte verticale $\sigma_{2}=\frac{1}{2} \gamma z$ a tne valeur inférieure au poins de couverture [a allégement $n$ ], indépendante de l'inclinaisory i. Le scalaire $k_{\text {g }}$ vaut cot j (pour $\mathrm{i}=45^{\circ}$, le tenseur est isotrope)

\section{b) Solutions plastiques}

Le champ de Sokolovsk (1965) comporte trois zones (deux rectilignes de Rankine et une curviligne) avec raccoldement selon une ligne de glissement. Sur la verticale $\mathrm{Oz}_{2}$ isostatique : $\sigma_{*}=$ arz Lorsque i croit de 0 à $\varphi$, pour $\phi=30^{\circ}$, a, coefficient d'allègement $\%$, varie de 1 à 0,8 . Bien sûr ici : $k_{0}=k_{3}$

Dièdre symétrique d'angle $>\pi$, à flancs inclinés à i ("vallée " Fig. 5b):

Une solution élastoplastique est proposée par Sokolovski (1965). La zone centrale est en régime élastiçue entourée de deux zones de Rankine.

Sur l'axe de symétrie z (isostatique ), $\sigma_{2}=$ ay z et le scalaire $k_{0}$ varient avec l'inclinaison l. Par exemple. pour un angle de frottement $\varphi$ de $30^{\circ}$, lorsque i décroît cle $\phi$ à 0 , a w coefficient d'alourdissement $n$, varie de 1.8 a 1. Le scalaire $k_{0}$ varie de 0,6 à 0,33 .

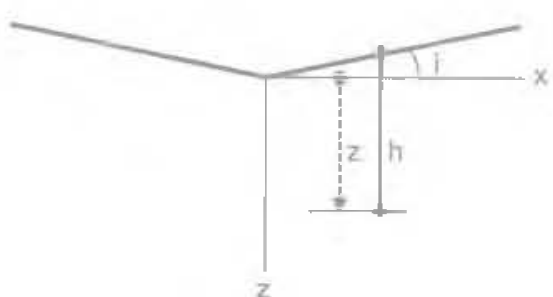

(b)

a) Dièdre d'angle < $\pi$; Dièdre d'angle $>\pi$.

Suriace with dihedral relief. a) Dihedral angle $<\pi$; b) Dihedral angle $>\pi$. 


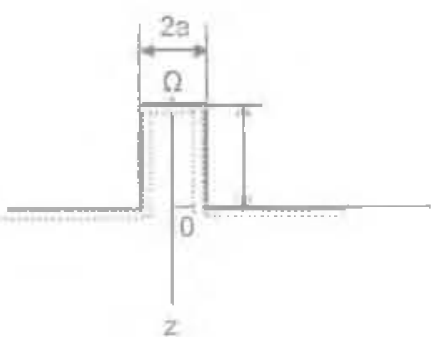

(a)

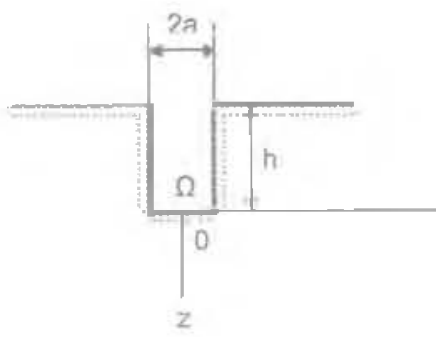

(b)

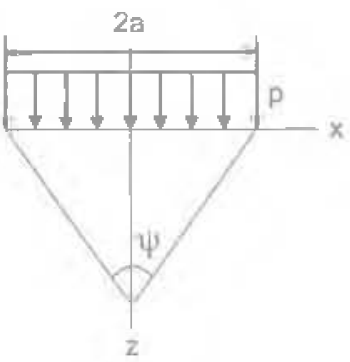

(c)

Fit. 6 Reliefs à profil rectangulaire (éperon et vallée en U).

a) Montagne (éperon) ; b) Vallée en U ; c) Champ de Boussinesq : charge untóforme.

Surface with rectangular relief.

a) Steep mountain ; b] L] shape valkey ; cl Boussinesc mode] (uniform loadf).

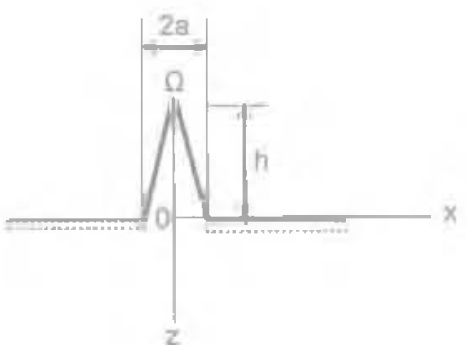

(a)

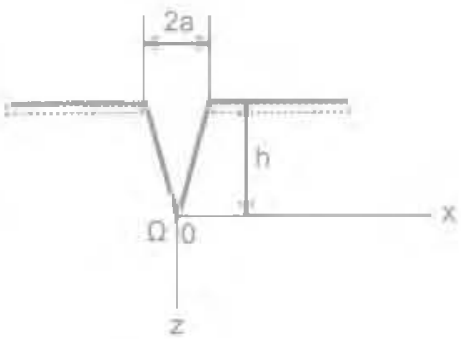

(b)

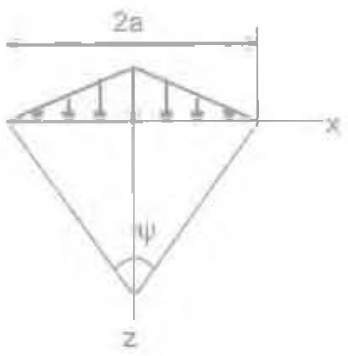

(c)

คG. 7 Reliefs à profil triangulaire (aiguille et vallée en $\mathrm{V}$

a) Montagne en aiguille ; b) Vallée en $V$; c) Champ de Boussinesq : charge triangulaire.

Surtace with triangular reliet"

a) Sharp mountain ; b) V shape valley; ; $\mathrm{c}$ Boussinesc model (triangula" load).

\section{4}

\section{Surface topographique à profil rectangulaire ou triangulaire (étude originale en élasticité)}

L'analyse proposée s'appuie, en supposant que la base du profil est une isostatique, sur le principe de substitution (pression p substituée au matétiau) et sur la théorie de Boussinesq. Elle permet l'étude, en régime élastique, de l'infuence de la topographie sur le champ $\left(\Sigma_{n}\right)$ : notamment tes effets sur la notion de " poids de couverture a de la présence d"un éperon rocheux [« pain de sucre w, " aiguille n] ou d'une vallée (a glaciaire nen U, a fluviatile v en V). En toute rigueur. l'application du principe de substitution supposeratt ic la verticalité d'une direction principale, ce qui n'est pas partout vérifié en cas d’un profil triangulaire (aiguile ou V).

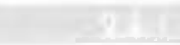

\section{Profil rectangulaire}

L'analyse comsiste, sur l'axe x (base du rectangle de hauteur h) à ajouter au champ initial (dont la contrainte verticale en fonction de $z$ est $\sigma_{1}^{\prime}=\sigma^{\prime}=\gamma(h+z)$. les effets d'une surcharge uniforme de valeur p sur une longueur 2 a représentant l'éperon rocheux lou la vallée) à la côte $z=0$ (Flg. 6 ).

Pour un tel massif semi infini horizontal, soumis a une surcharge (pour une montagne) ou à une traction (pour une vallée), verticale uniforme d'intensité p, sur une largeur 2 a, le champ de Boussinesq donnant les

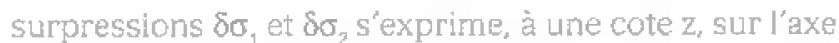
de symétrie $\mathrm{Oz}$ par :

$$
\begin{aligned}
& \delta \sigma_{1}=\frac{p}{\pi}(\psi+\sin \psi) \\
& \delta \sigma_{2}=\frac{p}{\pi}(\psi-\sin \psi)
\end{aligned} \quad \text { avec } \psi=2 \operatorname{arctg} \frac{a}{z}
$$

Selon le principe de substitution, d'un côté d'une surface isostatique, le matériau est remplacé par une pression nomale égale à la contrainte principale correspondante.

Pour l'éperon : $p=\gamma$ h (surcharge) ; pour la vallée $p=-\gamma$ h (traction).

\section{a) Pain de sucre (Fig. 6a)}

La surpression due à la surcharqe $p=\gamma$ h, appliquée à la coste $z=0$, est: $\delta \sigma_{1}=\gamma$ h $F$ avec $; F=\frac{(\psi+\sin \psi)}{\pi}$ sur l'axe z : $\sigma_{1}=\sigma_{x}$

Par superposition, la contrainte verticale totale sous l'éperon à la profondeur z est : $\sigma_{z}=\gamma z+\gamma \mathrm{hF}$

La contrainte verticale sous l'éperon est ainsi toujours plus faible que celle correspondant à un terrain semi infini de surface horizontale passant par be somrnet de l'éperon (point de référence), la différence étant : $\Delta \sigma=(\gamma z+\gamma \mathrm{hF})-\gamma(\mathrm{h}+\mathrm{z})$ soit $\Delta \sigma=\gamma \mathrm{h}(\mathrm{F}-1)<0$, qui correspond à un allègement relatif (déficit de $\sigma_{7}$ ) par ailleurs constaté par les mesures (voir \$ 4.2).

b) Vallée glaciaire (Fig. 6b)

La décharge correspondant à $\mathrm{p}=-\gamma \mathrm{h}$, appliquée à la cote $z=0$, est : $\gamma \sigma_{1}=-\gamma \mathrm{hF}$. La contrainte verticale 
totale sous ia vallée à une profondeur z est : $\sigma-\gamma(z+$ h) $-y$ hF, soit $\sigma=\gamma z+\gamma h(1-F)$. La contrainte verticale sous la valleee est ainsi toujours plus grande que celle due à un terrain semi infini de surface horizontale passant par la base de la vallée ; la différence étant: $\Delta \sigma=\gamma z-\gamma \ln (1-E)-\gamma z$ soit $\Delta \sigma=\gamma h(1-F)>0$, qui exprime l'alourdissement relatif (exces de o) constaté par les mesures (voir 34.2 )

\section{Surface topographique à profil triangulaire (Fig. 7)}

Des calculs analogues, effectués en considérant un profil triangulaire simulant une aiguille ou une valIée en V (Fig. 7a et 7b) aboutissent, en donnant à $\mathrm{F}$ la valeur $\frac{\psi}{\pi}$, à la mêne expression des resultats et aux mêmes conclusions.

\section{3}

\section{Modèles numériques}

Les méthodes numéniques (2D et $3 \mathrm{D}$ ) permettent de modéliser le champ de contraintes sous des reliefs quelconques, avec des structures et propriétés de terrrain et des condítions aux limites variées. Si elles sont possibles depuis longtemps (voir Goodman, 1989), les exemples de calcul restent copondant peu nombreux cans la bibliographie.

\section{1}

\section{But et hypothèses des modélisations effectués}

Les simulations numériques sont faites pour un massif ả topographie non plane en fonction des contraintes horizontales appliquées sur le bord d’un modêle-2D et traitées par la méthode des différences finies avec le logiciel FAC.

Le massif rocheux, homogène, élastique, jsotrope a pou caractéristiques

$$
\text { - poids volumique } \gamma=\rho \mathrm{g}=27 \mathrm{kN} / \mathrm{m}^{3} \text {; }
$$$$
\text { coefficient de Poisson } v=0,25 \text {; }
$$

- module d"Young E (paramètre d'entrée obligatoire pour la méthode numérique utilisée, sans influence sur les contraintes calculées) fixé arbitrairement à $15 \mathrm{GPa}$.

Les dimensions choisies (profondeur de $5 \mathrm{~km}$. largeur de 8 a $10 \mathrm{~km}$ ) permettent de s'affranchir des effets de bord, la zone d"étude étant airsi éloignée de plusieur's kilomètres de chaque bord. Le choix d'une montagne de $1000 \mathrm{~m}$ de haut et d'une vallée de $1000 \mathrm{~m}$ de profondeur (versant de $2000 \mathrm{~m}$ ) correspond à une situation moyenne rencontrée dans les Alpes.
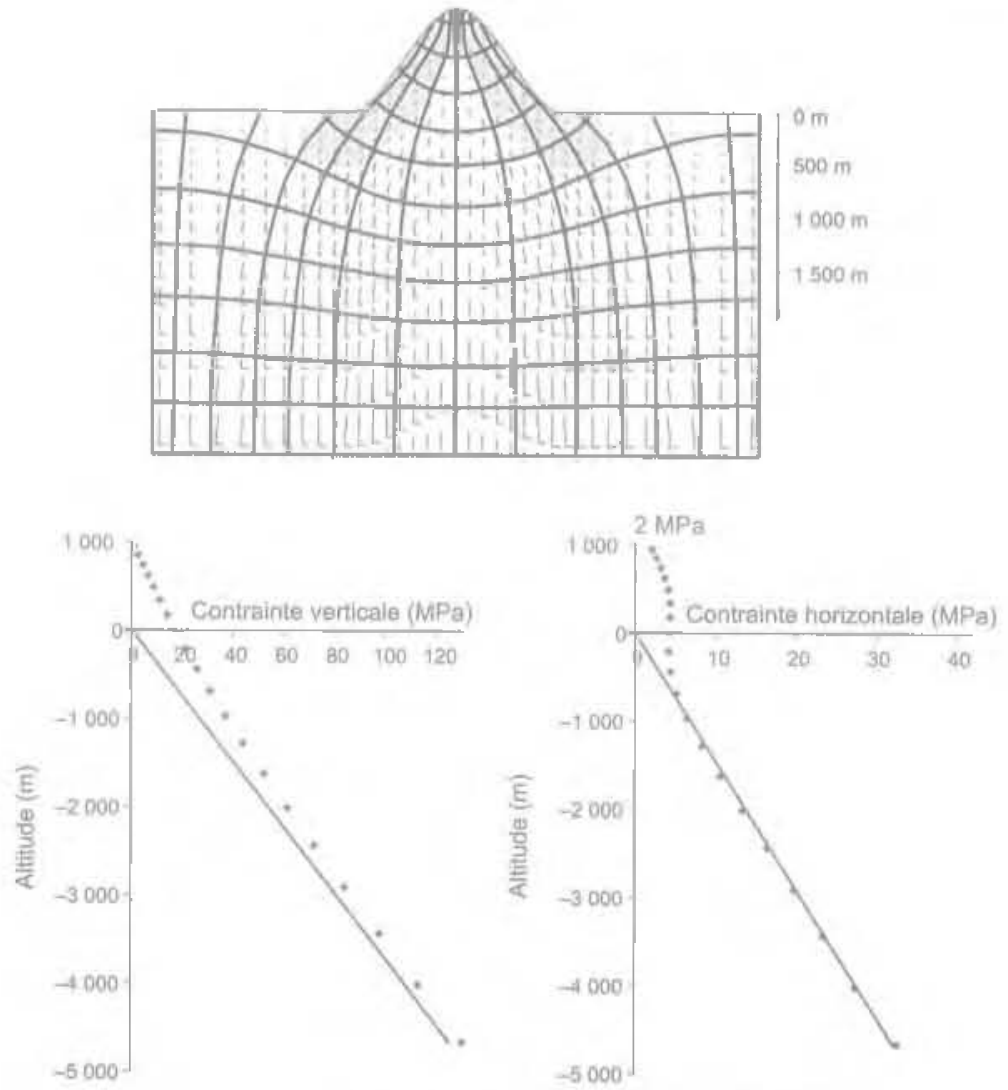

- Contraintes calculées saus l'axe de la montagne

- Contraintes obtenues en l'absence de montagne

F5. 5 Montagne isolée en condition lithostatique.

a) Isostatiques majeure $X$ et mineure $Y$ (zone grisée : inclinaison de $X$ supérieure à $15^{\circ}$ );

b) Valeurs des contraintes verticale o et horizontale $\sigma_{H}$ surr l'axe de la montagne.

Isolated mountain under lithostatic conditions.

a] Major $X$ and minor $Y$ isostatic lines (zones where slant of $X$ is $>15^{\circ}$ appear in grey).

b) Vertical stress $\sigma$, and horizontal stress $\sigma_{1}$ along mountain axis. 
Les conrtitions aux limites des modèles snnt:

- sur le fond horizontal : contraintes verticales constantes, équilibrant le poids total du modèle:

- sur la face supérieure et sur les deux faces verticales avant et arrière: contraintes nulles; ces hypothèses simplificatrices en contraintes planes ont été justifiées par Mayeur (1999, p. 137 ; calculs p. 326-327), après comparaison des resultats obtenus avec celles des déformations planes : les contraintes horizontales et verticales calculées sont très peu différentes; seuls les déplacements sont modifiés sensiblement:

- sur les faces latérales, deux types de conditions sont appliquées, soit des déplacements horizontaux bloqués (condition a lithostatique » : équivalente à un $\mathrm{k}_{0}$ latêral

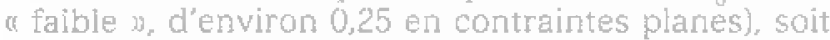
l'application d'une contrainte aux limites latérales $\sigma_{H}$ $=k_{0}-\gamma z$ (condition u tectonique $x$ ), avec trois valeurs Étudiées ici : $k_{\text {ka }}=0,5 ; 1 ; 1,5$, toutes trois supposant rester dans le domaine élastique $\left(\mathrm{k}_{\mathrm{a}}<\mathrm{k}_{0, a}<\mathrm{k}_{\mathrm{p}}\right)$.

\section{9}

\section{Effets de la topographie en condition lithostatique}

- sur les directinns principales du champ res contraintes (isostatiques de la figure 8a) qui sont modifiées surtout sous les flancs de la montagne, où la direction. principale majeure a tendance à s'aligner avec la ligne de plus grande pente. Létendue de la zone perturbée (dans laquelle la direction principale majeure s'écarte de plus de $15^{\circ}$ de la verticalel apparaît en grisè. Avec ce critère de $15^{\circ}$, la zone perturbée occupe une partie importante de la montagne à l'exception du centre où, par raison de symétrie, les directions principales sont l'horizontale et la verticale ; elle s'étend jusqu'à une nrofonderr de fon m snus le riveatl des plateaux, et latéralement au pied de la montagne sur une largeur comparable :

- sur les valeurs des contraintes principales verticale o, et horizontale $\sigma_{H}$ selon l'axe de la montagne (FIg. 8b). La contrainte horizontale est plus forte sous la montagne que sous les plateaux latéraux (droite de référence) : en sommet de montagne (ên concordance avec le calcul analytique, une compression horizontale non nulle, de $2 \mathrm{MPa}$ est obtenued, puis jusqu"à une profondeur de $800 \mathrm{~m}$. La situation s’inverse très légèrement au-delă,

\section{Cas d'une vallée isolée (Fig, 9)}

Les zones oủ les isostatiques sont les plus déviées. (Fig. 9a) se trouvent sous les flancs de la val]ée où, comme dans le cas de la montagne, la direction princjpale majeure tend à śorienter parailèlement à la ligne

La modélisation permet d'examiner les perturbations apportées par la présence de la montagne:
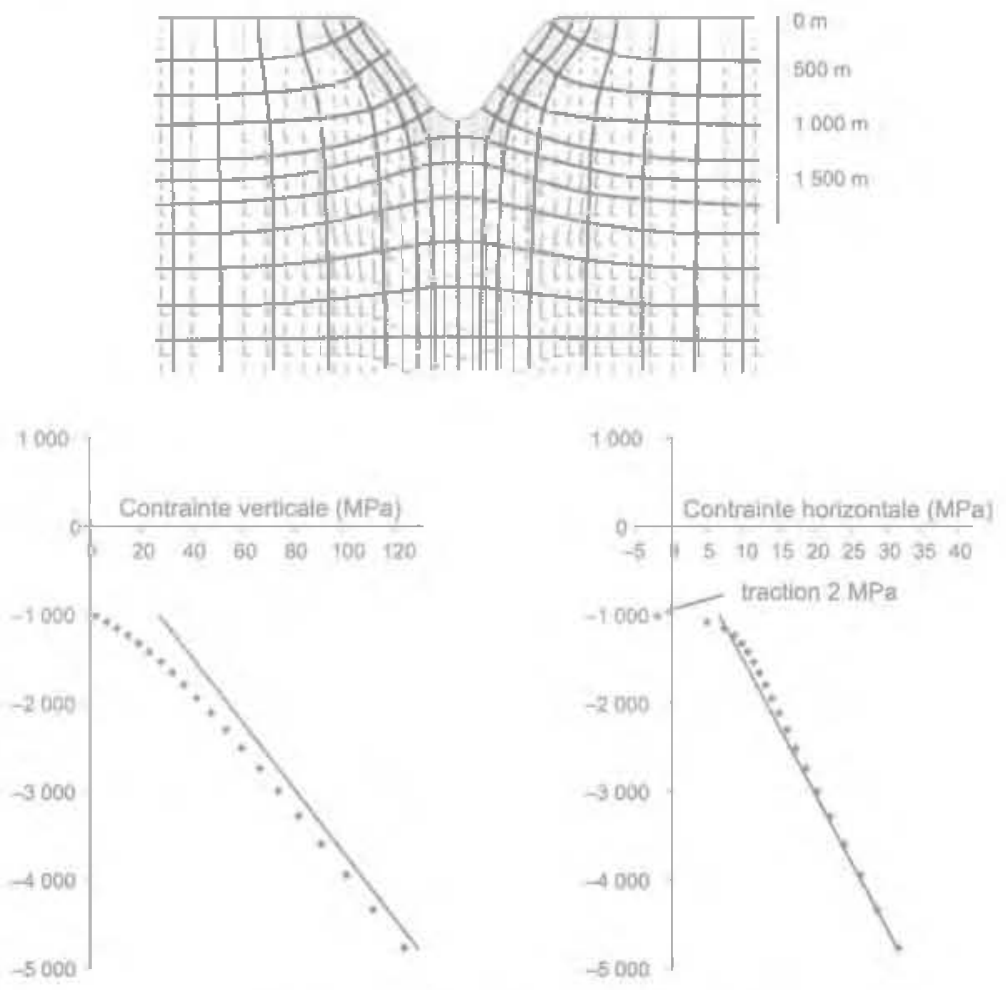

- Contraintes calculées sous l'axe de la vallée.

- Contraintes obtenues en l'absence de vallèe

FG. 9 Vallée isolée en condition lithostatique.

a) Isostatiques majeure $\mathrm{X}$ et mineure $\mathrm{Y}$ (zone grisée : inclinaison de $\mathrm{X}$ supérieure à $15^{\circ}$ ) ;

b) Valeurs des contraintes verticale $\sigma_{y}$ et horizontale $\sigma_{H}$ sur l'axe de la vallée.

Isolated valley under lithostatic conditions.

a) Major $X$ and minor $Y$ isostatic lines (zones where slant of $X$ is $>15^{\circ}$ appear in grey) :

b) vertical stress $\sigma$ and horizontal stress $\sigma_{4}$ along valiey axis. 
de plus grande pente. Ces zones d'épaisscur voisine do $500 \mathrm{~m}$ sont limitées à une profondeur d'environ $250 \mathrm{~m}$ sous le niveau du fond de la vallée.

Ies valeurs des contraintes verticale $\sigma$, et horizontale $\sigma_{H}$ selon l'axe de symétrie montrent des résultats " inversés y par rapport à ceux pour une montagne (Fig. 9b) : la contrainte horizontale $\sigma_{\mathrm{H}}$ est plus faible sous la vallée que sous les plateaux latéraux, jusqu'à une profondeur de $200 \mathrm{~m}$ sous le fond de vallée, puis la situation s'inverse. Juste sous le fond de vallée. conformément au calcul analytique, une petite zone est en traction $\left(\sigma_{\text {r: }}=-2 \mathrm{MPa}\right)$,

\section{3}

\section{Influence de la tectonique (Figs. 10 et 11)}

Pour simuler des conditions tectoniques existant dans les chaines de montagne, une approche dynamique est possible (Savage et al., 1992). Elle nécessite den donnces róalistes sur los teux de déformation et l'histoire de la chaîne [surrection, creusement des vallées.... .

L'effet d'un serrage latéral ou d'une extension peuvent être étudiés plus simplement de manière statique, par application du principe de superposition. (Timoshenko et Goodier, 1970) en additionnant au champ gravitaire celui résultant d'un cas de chargement latéral sur un milieu non pesant.

Dans le cas d'un modèle ane montagne-une valm lée $n$, la figure t0 montre, en fonction de l'intensité de la contrainte tectonique latérale appliquée $\sigma_{\mathrm{H}}=\mathrm{k}_{\mathrm{b}} \gamma \mathrm{z}$. l'évolution des contraintes verticale et horizontale (voi. sines des contraintes principales) sur l'axe de la monta. gne et sur l'axe de la vallée.

La variation de $\sigma_{\text {a }}$ avec l'augmentation de $k_{\text {los }}$ est à peine sensible sous la montagne, mais déja plus nette sous la vallée où o augmente avec $k_{0 x}$ jusqu'à erviron $2000 \mathrm{~m}$ sous le fond de vallée. La variation relative est faible sauf à proximité du fond de vallée : à $150 \mathrm{~m}$ sous
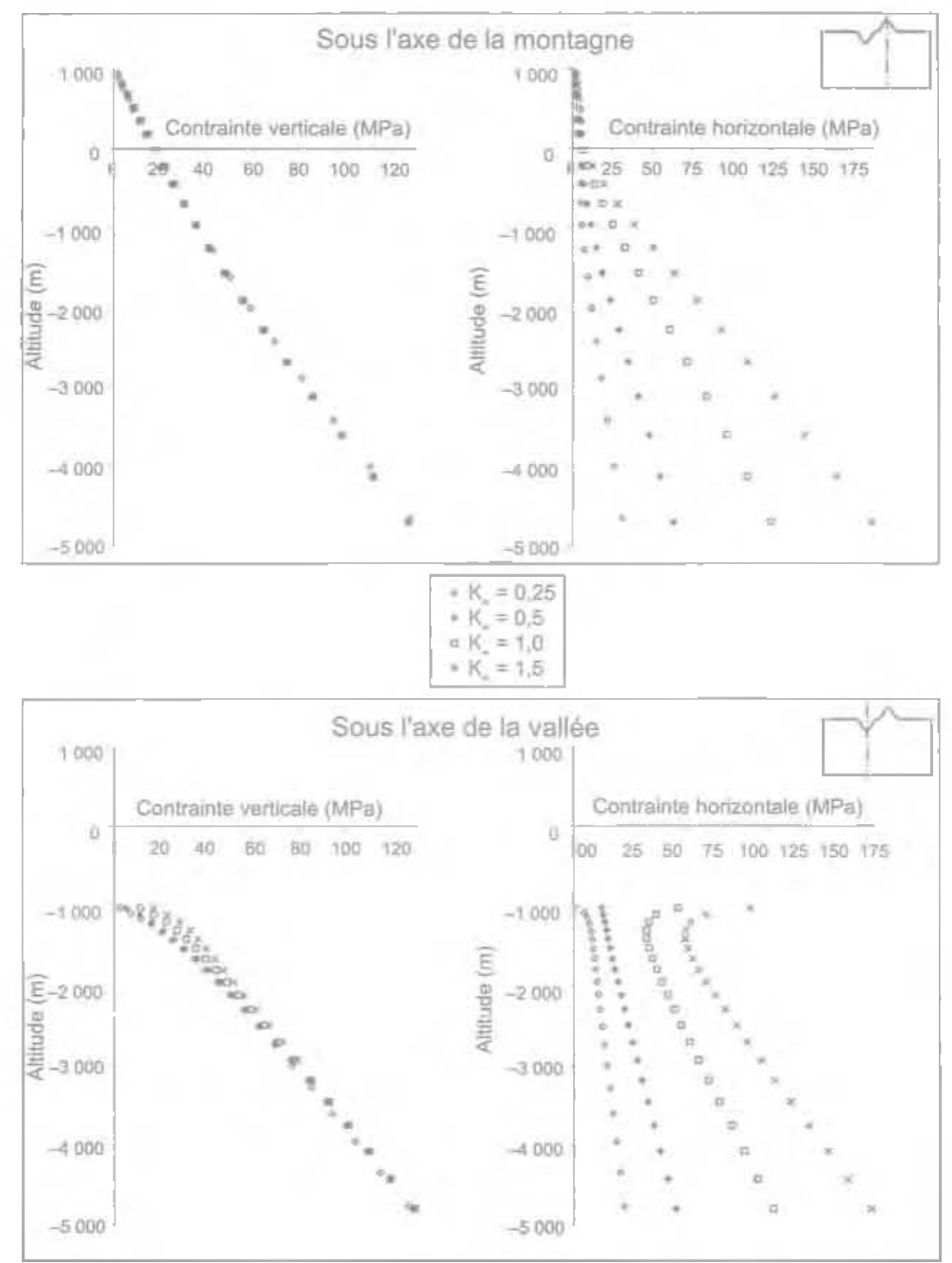

Fig. 10 Effets des conditions tectoniques latérales : valeurs des contraintes verticale et horizontale sous la montagne

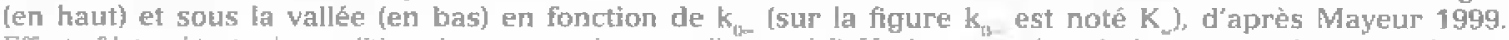
Effect of laterai tectonic conditions (one mountain-one valley model). Horizontal and vertical stresses under mountain axis (upper parti and valley axis [lower part) with different values of $\mathrm{K}_{\mathrm{w}}$ i= $\mathrm{k}_{\mathrm{fl}}$ value applied on lateral limits of the model], after Mayeus 1999 

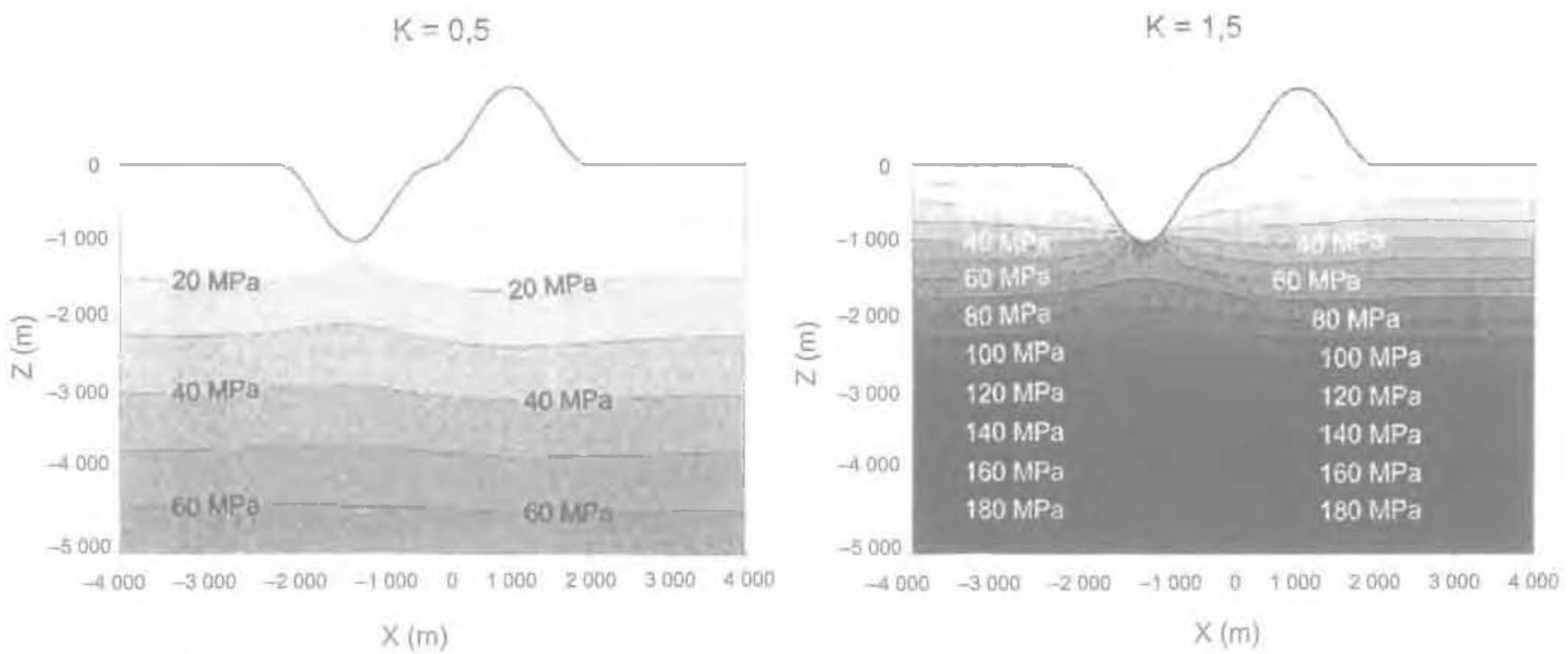

Fig. 11 Effets des conditions tectonjques latérales sur les contraintes horizontales : isovaleurs des contraintes horizontales pour deux situations de serrage latéral ( $\mathrm{k}_{\mathrm{kn}}=0$, 5 à gauché; $\mathrm{k}_{\mathrm{gr}}=1,5$ à droite), d'après Fabre et al., 2002. Effect of lateral tectoric conditions (one mountain-one valley nodel) on horizontal stress values : isovalues for 2 situations of latera] conditions ( $K=k_{-}=0.5$ at left $; ~ K=k_{t-}=1.5$ at right), after Fabre at al. 2002 .

le fond de la vallée, la contrainte verticale pour $k_{0-}=1,5$ est le double de ceille obtenue pour $\mathrm{k}_{\mathrm{am}}=0,5$.

L'augrnentation avec $\mathrm{k}_{\text {no }}$ des contraintes horizontales calculées est beaucoup plus importante; elle est particulièrement nette sous le fond de vallée où la concentration de contrainte est très forte. Un changement de forme des courbes est observé entre le cas de serrage faible fou la contrainte horizontale reste nulle en surface) et les cas de serrage plus marqué, avec création d'une zone de surface en a surcompression m.

Pour le même modèle une montagne-une vallée m, la figure 11 montre les isovaleurs des contraintes horizontales pour deux valeurs du coefficient $k_{m}: k_{n=-}=0,5$ valeur classigue a faible $0 ; k_{0 x}=1,5$ valeur aforte $:$ correspondant à un serrage lateral bien marqué. La vallée concentre les contraintes horizontales : on atteint $\sigma_{k:}=$ $60 \mathrm{MPa}$ sous la vallëe à $500 \mathrm{~m}$ de profondeur dans le deuxième cas, ce qui correspond à un rapport $\sigma_{\mathrm{H}} / \sigma_{\mathrm{v}}$ local de 2,2.

\section{4}

\section{Effets de la topographie et des conditions tectoniques}

\section{Sur l'orientation des directions principales}

Les orientations des directions principales sont fortement déperdantes des contraintes tectoniques

- lorsque $k_{0}<1$. la direction principale majeure est Subverticale (avec une tolérance de $15^{\circ}$ ) dans la majeure partie du massif, sauf dans une bande parallèle à la surface topographique dont l'épaisseur depend de $\mathrm{k}_{(\mathrm{k} s}$

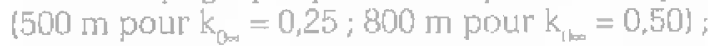

- lorsque $k_{\text {tle }}=1$, les orientations sont très dispersées, car mal définies en raison de la cuasi-isotropie du champ de contraintes:

- Iorsque $\mathrm{k}_{\mathrm{m}}>1$, la direction principale majeure est subhorizontale (avec une tolérance de $15^{\circ}$ ) dars une grande partie du massif, sauf dans une zone aplatie horizontalement qui tangente le fond de vallée et englobe presque tout le volume de la montagne; en dessous du fond de vallée, les directions principales se rapprochent de l'horizontale et de la verticale.

La situation est différente ä proximité du versant, tes modélisations confirment que la direction principale majeure tend à s'orienter parallèlement à ligne de plus grande pente, ce qui rejoint l'obsenvation de Talobre (1967) et diffère du résultat des calculs analytiques pour un versant infini (S 2.2).

\section{Sur la valeur des contraintes principales}

En condition a lithostatique n ( $\mathrm{k}$, faible), les écarts entre la contrainte horizontale (resp. verticalel calculée sous une vallée et la valeur de rétérence en l'absence de relief (" poids de couverture $)$ ) diminuent avec la profondeur: de $19 \%$ (resp. $40 \%$ ) a $500 \mathrm{~m}$ sous le fond de vallée, à $14 \%$ (resp. $17 \%$ ) à $1000 \mathrm{~m}$, et à $9 \%$ (resp. $8 \%$ ) à $1500 \mathrm{~m}$.

Lapplication de contraintes tectoniques laterales modifie surtout les valeurs des contraintes horizontales, qui augmentent en généra! proportionnellement aux contraintes imposées aux limites, sauf sous les vallées, ou elles sont plus fortement accrues (" effet de concentration ). et sous les montagnes, où elles sont un peu atténuées. Les contraintes verticales ne sont que peu modifiẻes, mais leurs modifications sont significatives: elles diminuent légèrement sous les montagnes ( $₫$ effet d'allégement n) et augmentent plus fortement sous les vallées ( $($ effet d'alourdissement $n$ ), en accord qualitatif avec les calculs analytiques. 


\section{Retours d'expérience}

Des exemples naturels, dans des terrains et des conditions tectoniques variées (Est de la France, Alpes) illustrent le caractère tensoriel de ( $\left.\mathbf{K}_{\mathbf{p}}\right)$, soulignant en particulier l'hétérogénéité et l'anisotropie des contraintes horizontales et les effets d'alfégement ou d'alourdissement de $\sigma_{y}$ liés aux relíefs.

\section{1}

\section{Est de la France (zone à relief modéré dans un contexte " en extension ")}

Les mesures de Froidevaux et al. (1980) en France et en Baviére et la carte européenne des contraintes, établie à l'aide de donnés géopliysiçues (Müller et al., 1992). montrent géréralement une forte anisotropie des contraintes dans le plan horizontal, avec parfois la contrainte principale mineure en traction ( $\sigma_{\text {hit }}$ négatif').

Trois sites français ayant donné lieu à des observations (Vieux Pré) è à des mesures (Soultz, Bure) sont analysés ci-après.

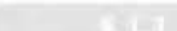

\section{Secteur du barrage EDF de Vieux Pré}

Lors des injections réalisées sous le barrage, la surprise a été d'observer de très importantes absorptions de coulis de ciment dans le substrat (grès vosgiens du Trias inférieur], notamment dans sa partie profonde (à une cote inférieure au fond de la vallêl, alors que la fracturation générale était considérée comme faible et les fractures non ouvertes en profondeur. Lrinterprétation a été donnée (Haguenauer et Hily, 1987) par la présence d'une famille de fissures u ouvertes in perpendiculaires à h (ou // à $\mathrm{H}$ ), orientée $\mathrm{N}-\mathrm{S}$, et considérée comme une comséquence de la u distension a régionale (w rift rhènan $v$ ).

Linfluence des contraintes naturelles sur les capacités d'injection du massif rocheux pour un site de barrage n'est pas une observation nouvelle: Antoine et Fabre (1979) l'avaient signalée, majs en situation inverse au barrage de Vinça, où l'absorption était anormalement fable en regard dune fracturation très dense.

\section{Site de Soultz-sous-Forêts}

Dans le cadre d"un projet européen de géothermie à Soultz-sous-Forêts, de nombreuses mesures de contraintes ont été réalisées par la méthode HTPF (Hydraulic Tests on Preexisting Fractures, Cornet et Valette. 1984) et analysées (Cornet et al., 2006). Le tableau I résume les principaux résultats acçuis entre 1400 et $5000 \mathrm{~m}$ de profondeur.

Globalement, l'état de contraintes est anisotrope avec deux contraintes principales voisines du poids des terres (isotropie transversale, $\sigma_{f /} / \sigma_{y}=1$ ) et la contrainte $\sigma_{h}$ plutôt faible $\left(\sigma_{h} / \sigma_{v}=0,54\right)$. Concernant l'orientation du tenseur $\left(\Sigma_{0}\right)$, il est suggéré une légère rotation de la direction principale majeure horizontale $\mathrm{H}$ entre $\mathrm{N} 165$ à $1600 \mathrm{~m}$ de profondeur et N 185 à $3800 \mathrm{~m}$, en accord

\begin{tabular}{|c|c|c|c|}
\hline TABLEAU I & $\begin{array}{l}\text { eurs des c } \\
\text { s-Forêts } \\
\text { ss values at }\end{array}$ & $\begin{array}{l}\text { aintes } \\
\text { ès Co } \\
\text { z-sous- }\end{array}$ & $\begin{array}{l}\text { rées à Soultz } \\
\text { at al., } 2000 \mathrm{f}\end{array}$ \\
\hline Protondeur $[\mathrm{m}$ ) & on (MPa) & chin/ov & oH/ON \\
\hline 1980 & 26,3 & 0.535 & \multirow{2}{*}{$\begin{array}{c}\text { légèrement } \\
>1\end{array}$} \\
\hline 2880 & 39.5 & 0,548 & \\
\hline 3315 & 45 & 0,541 & \multirow{2}{*}{$\begin{array}{l}\text { légèrement } \\
<1\end{array}$} \\
\hline 4550 & & 0,537 & \\
\hline
\end{tabular}

avec des données géophysiques et des mesures sur des sites voisins en Allemagne (Bad Urach et KTB, in Comet et al, 2006).

Plusieurs auteurs (Rummel et Baumgartner, 1991 ; Heinemann, 1994...) ont proposé pour ce site une expression des contraintes principales $\sigma_{h}, \sigma_{H}$ et $\sigma_{y}$ en fonction de la profondeur pour les mesures au-delà de $1400 \mathrm{~m}$ (changement important dans la géologie ả 1377 m), notamment Cornet ef al. (2006) :

$\sigma_{\mathrm{h}}=0,54[33,8+0,0255(z-1377)]\left(\mathrm{MPa}_{\text {, avec } z \text { en } \mathrm{m}]}\right.$

Avec cette formule, le calcul pour la surface $(z=0)$ conduirait à une valeur faiblement négative $\left(\sigma_{\mathrm{h} 0}=-0,7 \mathrm{MPa}\right)$. en accord avec le contexte extensif reconnu (graben rhénan). Un résultat similaire io en traction] serait obtenu sur un autre sike [rançals (Mern. fons, in Cornet et Burlet, 1992).

\section{Site de Bure}

Sur le site de Bure (laboratoire souterrain, projet ANDRA) des mesures de contratntes par stimulation hydraulique ont été effectuées en 2000 dans cinq forages entre 380 et $640 \mathrm{~m}$ de profondeur (Wileveau et al., 2006\%.

Les equations des contraintes en fonction de la profondeur ont été donnèes par Gunzburger et al. (2007). Celles-ci sont đifférentes pour les formations calcaires [Oxfordien jusqu'áa $400 \mathrm{~m}$ et Dogger à partir de $550 \mathrm{~m}$ ]) et pour les argilites ( 420 à $550 \mathrm{~m}$ ) :

- pour les calcaines: $\sigma_{\mathrm{h}}=0,710^{-3} \mathrm{z}+7,9$ et $\sigma_{H}=0,710^{-3}+13.9(\mathrm{MPa}$, avec $z$ en $\mathrm{mil}$;

- pour les argilites : $\sigma_{1}=6,810^{-3} \mathrm{z}+9,1$ et $\sigma_{H}=6,810^{-3}+11$ ( $(\mathrm{MPa}$, avec $z$ en $\mathrm{m}$ ).

De ces équations, sont déduits : pour les calcaires $\mathrm{k}_{0+1}=\mathrm{k}_{\mathrm{m} !}=0,03$; et, pour l'argilite $\mathrm{k}_{0 \mathrm{~h}}=\mathrm{k}_{0 \mathrm{~kL}}=0,28$.

En dehors de souligner l'influence de la lithologie (propriétés mécariques). la singularité des mesures faites à Bure est de donrer de très faibles valeurs pour les gradients de contraintes horizontales $k_{\text {oh }}$ et $k_{0 \mid-1}$ : l'extrapolation en surface donne des valeurs de contraintes positives assez fortes. Cependant, l'orientation trouvée pour la direction principale majeure dans ce secteur (N 155) est en bon accord avec les déterminations faites sur les deux sites précédents.

\section{2}

\section{Alpes françaises (zone de fort relief dans un contexte " en compression ")}

Une importante campagne de mesures par la méthode HTPF a été réalisée entre 1990 et 2000 pour le projel de tunnel de base franco-italien " Maurienne- 
TaBlfau ॥ Résultatts de la détermination du tenseur des contraintes pour 16 forages profonds (reconnaissances du tunnel de base Maurienne-Ambin) (voir les domaines Fig. 12).

p et $\mathrm{z}$ : profondeur et altitude de la zone de mesıre : $y$ h : poids des terres (calculé ; h peut différer de p pour un forage incijné) : $\sigma_{4}$ : valeur de la contrainte verficale mesurèe; $\sigma_{\mathrm{h}}$ (resp. $\sigma_{\mathrm{H}}$ ) : valeur de la contrainte horizontale minewre (resp. majeure) mesurée; l'azimut $\alpha$ de H est précisé entre parenthèses.

Stress tensor determined in 16 boreholes (HIPF method) near Modane (Maurienne-Ambir tunnel project) (see geographic domaines on Fig. 12).

$p$ : depth : $z$ : altitude ; $\gamma$ h : overbunder pressure; $\sigma_{y}$ : vertical stress ; $\sigma_{t_{2}}$ : horizontal minor stress; $\sigma_{H}$ : horizontal major stress : o arimut of $\mathrm{H}$ (berween brackets].

\begin{tabular}{|c|c|c|c|c|c|c|c|}
\hline Domaine & Forage & $p(m)$ & $2(\mathrm{~m})$ & $\gamma h(\mathrm{MPa})$ & $\sigma_{v}(\mathrm{MPa})$ & $\sigma_{\mu}\left(\mathrm{MPa}_{\mathrm{a}}\right)$ & $\sigma_{31}\left(\mathrm{MP}_{a}\right)$ \\
\hline $\mathrm{A}$ & $\$ 5$ & 740 & 780 & 20 & 19 & 10 & $30[N 53$ \\
\hline $\mathrm{A}$ & $\mathrm{S} 23$ & 473 & 569 & 13 & 14 & 7 & 9 (N58) \\
\hline$B$ & F16 & 1141 & 840 & 29 & 23 & 28 & 36 (N126) \\
\hline $\mathrm{B}$ & 54 & 535 & 624 & 21 & 17 & 20 & 30 (N53) \\
\hline $\mathrm{B}^{\prime}$ & $\mathrm{Fr}$ & 1099 & 753 & 29,7 & 21.4 & 20.2 & 23.5 (NG7) \\
\hline $\mathrm{C}$ & F5 & 161 & 894 & 4,3 & 8,7 & 5.3 & $8,5[N 172]$ \\
\hline C & F8 & 391 & 839 & 10,6 & 15,3 & 7.2 & $13.5(N 145)$ \\
\hline $\mathrm{C}$ & $\mathrm{F} 17$ & 516 & 735 & 13,9 & 18,1 & 8,5 & $13,6(\mathrm{~N} 181)$ \\
\hline $\mathrm{C}$ & F31 & 912 & 864 & 24,6 & 37,4 & 8,1 & 22,8 (N133) \\
\hline $\mathrm{C}$ & $\mathrm{F} 33$ & 408 & 751 & 11,0 & 15,5 & 15,3 & 28,3 (N123) \\
\hline C & $\mathrm{F} 57$ & 414 & 831 & 11.2 & 12,0 & 10,0 & $15,4(N 211)$ \\
\hline C & $\mathrm{FG7}$ & 1150 & 675 & 30 & 25.2 & 13 & $28,8(N 157)$ \\
\hline $\mathrm{D}$ & $\mathrm{F} 14$ & 818 & 795 & 22,1 & 21,3 & 14,5 & $37,1(\mathrm{~N} 1)$ \\
\hline$D$ & $F 21$ & 709 & 824 & 19,1 & 20.4 & 10.4 & $17.1[\mathrm{~W} 11 \mathrm{~d}$ ] \\
\hline atyp. & Fbbis & 923 & 629 & 24,9 & 8.5 & 15,1 & $48,7(N 125)$ \\
\hline atyp. & F25 & 298 & 1018 & 8,0 & 3,0 & 3,9 & 12,8 (N45) \\
\hline
\end{tabular}

Ambin mouvelle ligne ferroviaire Lyon-Turin). Le tableau ll donne les résultats de la détemmation de $(\Sigma)$ pour 16 forages profonds. La verticalité d'une contrainte principale a été ici soit vérifiée (S23), soit adoptée comme hypothèse étant donnée la profondeur de la zone de mesure.

\section{Massif d'Ambin}

Deux domaines principaux A et B different par leur régime de contraintes (Fig. 12) :

- A : bordure externe (S5 et $\$ 23$ ), où règnerait un $\left(\Sigma_{0}\right)$ relativement simple avec $\sigma_{y}$ majeure et $\sigma_{H}, \sigma_{H}$ voising (orthotropie de révolution) et faibles $\left(k_{0}=0,5\right)$ :

- B : partie centrale du massif $(F 16, S 4)$ où la verticale est la direction de la contrainte mineure, les contraintes horizontales fortes $\left(\sigma_{\mathrm{H}} / \sigma_{\mathrm{v}}=1,6 ; \sigma_{\mathrm{h}} / \sigma_{\mathrm{v}}=1,2\right)$ et où l'azimut a de H semble encore, même a la profondeur importante des mesures, être influencé par le relief: $\mathrm{H}$ tend ainsi à se situer perpendiculairement aux vallons bien marqués existant en surface (val Clarea pour S4, ruisseau d'Ambin pour F16 J.

L'allègement de contrainte verticale constaté $(0$, $<\gamma$ h) est qualitativement en accord avec des solutions analytiques (\$ 2.3 ) et numériques (\$ 3.3.3).

\section{Valee de l'Arc \\ Vallée de l'Arc}

Le champ des contraintes est hĕtérogène. A côté de deux cas atypiques (très proches d'un accident géologique : F6bis et F25), trois domaines peuvent cependant être identifiés (Fig. 12) :

$-B^{\prime}=a ̀ 2.5$ kilomètres en rive gauclie ( $F 7$, sondage le plus au cosur du massif avec F16), les contraintes mesurées sont pratiquement isotropes et valent environ $75 \%$ de la contrainte lithostatigue (w effet d'allégement $n: \sigma_{2}<\gamma z$, comme dans le massif d'Ambinj;

- C : à moins d'un kilomètre du fond de vallée, et $300 \mathrm{~m}$ sous la cote de l'ArC, les contraintes horizontales sont très anisotropes (rapport $\sigma_{\mathrm{H}} / \sigma_{\mathrm{h}}$ jusqu'à 2,8) orientées approximativement parallelement (h) et perpendiculairement $(H)$ a la vallée: la contrainte verticale vaut entre 1,1 et 2 fois le poids des terres: " effet d'alourdissement $n\left(\sigma_{z}>\gamma z\right)$ bien marqué, dî au poids des versants:

- D : entre 1 et 2 kilomètres en rive droite, et toujours $300 \mathrm{~m}$ sous la cote de l'Arc (sondages F14 et F21), les contraintes horizontales sont ici encore anisotropes $\left[\sigma_{\mathrm{h}} /\right.$ $\sigma_{v}$ entre 0,5 et 0,$7 ; \sigma_{\mathrm{H}} / \sigma_{\mathrm{v}}$ entre 0,9 et 1,7 ) et la contrainte verticale est très proche du poids des terres. 


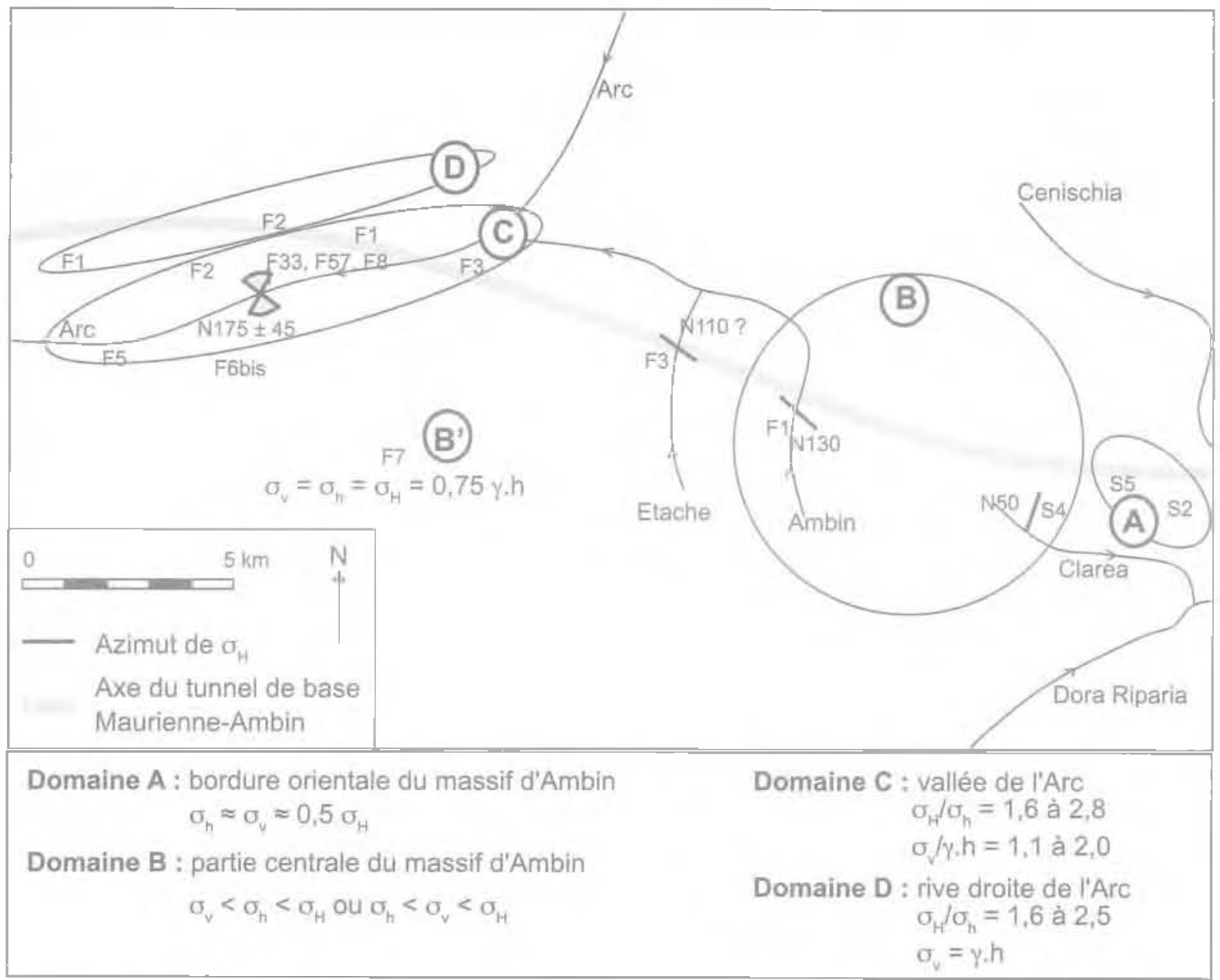

Fa. 12 Carte générale des contraintes dans les secteurs de Modane et du mont d'Ambin, d'après les mesures HTPF [1990-2000).

General map of stress near Modane and Mont d'Ambin (from HTPF measurements, 1999-2000). Four main domains of natural state of stress are deffned (see text), after Mayeur 1999.

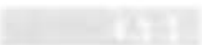

Bilan

En accord avec les solutions analytaues et les modélisations présentées, les mesures montrent systématiquement une concentration des contraintes horizontales sous bes vallées avec augmentation du coefficient $k_{0,}$, sous les vallées (Fabre et al., 2002). Cette situation amene à nuancer l'argument qui preconisait, dans les études préliminaires du projet de tunnel de base franco-italien, de suivre Ies vallées pour \& éviter les contraintes ». Dans le secteur de Modane, on se trouverait plutôt avec une contrainte principale majeure horizontale nettement supérieure au poids des terres et perpendiculaire au tunnel, situation confirmée par d'autres indices (discage de carottes, ovalisation de forages).

\section{5}

\section{Conclusion}

Le champ $\left\{\Sigma_{0}\right)$ est une caractéristique intrinseque des terrains, dont la détermination fait partie de la reconnaissance du milieu. Ses composantes interviennent dans la conception et la réalisetion d'ouvrages souterrains ou même de surface (barrages). Il est carac- térisé le plus souvent par un tenseur adimensionnel $\left(\mathbf{K}_{0}\right)$.

Dans le cas particulier d'un milieu pulverulent ả surface libre horizontale, il se ramène à un scalaìre urique $\left.k_{n} !=\sigma_{1}^{\prime} / \sigma^{\prime}\right)$ ou $k_{n j}(=v /[1-v)$ en élasticité

Dans les massifs rocheux et les milieux cohérents er genéral, deux caractéristiques doivent être prises en compte

- dune part, la non-nullité du champ en surface : $(\Sigma)$ a pour composentes $0, \sigma_{n 0}$ et $\sigma_{\text {Ho }}$ ce qui condujl à utiliser le gradient vertical et non le rapport des contraintes pour caractériser le champ ;

- d'autre part, une frécuente anisotrople de $\left[\Sigma_{\gamma}\right)$ daras le plan horizontal $\left(\sigma_{b} \neq \sigma_{00}\right)$, résultant de l'histoire géologique (deformation tectonique, érosion), qui conduit à deux gradients et à deux valeurs de $k$ distinctes pour les directions principales horizontales.

Sous un relief subhorizontal ou loin de la surface topographique, $\left[\Sigma_{n}\right]$ présente une direction principale verticale et une homogénéité dans le plan horizontal ; il se caractérise alors par cinq paramètres : un angle $\alpha$ [azimut de la direction $H$ ), deux coefficients $\mid k_{0 ! n}$ et $k_{\text {bit }}$ l et deux contraintes horizontaies de surface $\left(\sigma_{\mathrm{Ho}}\right.$ et $\left.\sigma_{\text {hio }}\right]$. Les valeurs de ces paramètres et notamment le classement des scalaires principaux $\left(\mathrm{k}_{0 \mathrm{H},} \mathrm{k}_{\mathrm{ap},}, 1\right) \mathrm{du}$ tenseur $\left(\mathrm{K}_{0}\right)$ sont nettement reljés à l'histoire des déformations tectoniques du secteur. 
Parfois, dans le cas de zomes en extension, la contrainte principale mineure de surface $\left(\sigma_{n g}\right)$ peut être une traction, comme dans l'Est du territoire français, entre le bassin parisien el le graben rhénan. N 65 à Bure, la direction de l'extension deviendrait pratiquement E-W dans la zone profonde des sondages de Soultz. Les mesures sur le site de Bure font apparaître une nette hétérogénéité du champ au niveau de la couche d'argilite (discontinuité de $\sigma_{\mathrm{b}}$ ) et de três faibles valeurs des coefficients $k_{\mathrm{ut}}$ et $\mathrm{k}_{\mathrm{ub}}$.

Dans le cas de relief accidenté, le champ n'est plus homogère dans le plan horizontal et la détermination des paramètres $\sigma_{H \mathrm{H}^{\circ}} \sigma_{\mathrm{HO}}$, $\mathrm{k}_{\mathrm{OH}}$ et $\mathrm{k}_{0 \mathrm{O}, \mathrm{s}}$ est déli- cate au voishage de la surface, sur une épaisceur évaluée à environ une fois la profondeur des aspérités du relief, d’après les modélisations effectuées (Mayeur, 1999). Les zones situées sous des fonds de vallée présentent fréquemment de fortes contraintes horizontales (direction $\mathrm{H}$ souvent perpendiculaire à l'axe cle la vallée et $\mathrm{k}_{\text {vH }}>1$ ) ; et, sous les versants. l'inclinalson des axes principaux peut devenir forte (une contrainte principale en surface parallèle au versant). Ces effets sont renforcés par le serrage tectonique, situation qui paraît être actuellement le cas dans la direction N 140 à N 180 pour la zone de la vallée de l'Arc près de Modene.

\section{$\overline{\text { Bibliographie }}$}

AFIES, Groupe de Trawaid no 1 - a Carac térisation des massifs rocheux utile à l'étude et à la réalisation des auwrages souterrains u. Tundels et Otrrages Sout terains, $\pi^{\circ} 177,2003, \mathrm{p} .138-186$.

Antoine P. Fabre D. - Réflexions sur le rôle et l'orientation des études de fractura. tion pour la fomdation d'un barrage an béton. Bul, AGI, n' 20, 1979, p. $40-44$

Burlet D., Fabre D., Ménard G. - Mesures de contraintes in situ par tests hydrauligues de fracture en forage, dans le cadre d"un projet de grand tumnel sous les Alpes. Proc gh int Congress ISRMt, Tokyo, Ba! kersa, wol. 3, 1995, p. 1141-1144.

Carpegna M., Mayeur B., Fabre D., Gaudin B. Toumery H. Bultel E - InFluences du relief et de la tectonique pour la préwision des états de contraintes sous fort recouvrement. Proc. gil int. Congress ISRM, Paris, Balkema, 1999, p. $113 \bar{\gamma}$. 1142 .

CFMR - Manuel de Mécanique des Roches, Presses de l"École des mines. Paris, 2000, tome 1, chapitre 7.

Comet F.H., Valette B. - In situ stress determination from hydrailic injection test data. J of Gsophys. Ries. vol. 89, 1984. p. $11527-11537$

Cornet F.H., Burbet D. - Stress field determimation in France by hydraulic tests in boreholes. J. of Geophys. Res.vol. 97, B8. 1992. p. 11829-11849.

Davis. Salvadurai - Elasticity and Geomechánics. Cambiridge Lniversity Press. 1996. $200 \mathrm{p}$

Desccudres F. - Mecanigue des roches Cours polycopie, LMR-ISRE, École polytechnicue féderale de Lausanne. 1984.
Falore D. Sirievs R Maveur B. - Pamaméires caractêrisant l'état de contraintes naturel dans les massifs rockeux. C.R. Symp. Int. PARAM 2002, Faramètres de calcul géotechnique, Presses de l"ENPC LCPC. Paris, 2002. D. 359-368.

Fairhurst C. - Stress estimation in rock : A brief history and review. Int. J'. of Rock Mech. \& Min. Sc. vol. 40, 2003, p. 957975

Froidevaux C. Paquin C., Souriau M. - Iectonic siresses in France : In situ measurements with a flat jack. J. of Geophys. Res., vol. 85, 811, 1980, p. 6342-6346

Goguel J. - Galcul des contraintes dans I'hypothese de la relaxálion complete. C. R. Acad. Sc. tome CCXN. 1942, p. 470.

Goodman R.L. - Introduction to rock mechanics. John Wiley \& sons, New York, 1989

Gunzburger Y. Comet. F.H. - Rhaologica: characterization of a sedirnentary formation from a stress profile inversion. Geophys. J. Int., voJ. 168, 2007, p. 402 418.

Haguenauer B.. Hilly I. - Maissance et déclin du bassin parisien dans ses mar ges orientales. Bull. Inform. Gëol. Bassin de Paris. Mém. h. -sér. 1706. p. 105-116.

Hoek E., Brown E.T. - Undergruma' excawations in rock. Institution of Mining and Metcilurgy, London. 1980, p. 93-101.

Sirieys P. - Structure, contraintes lithostatiques et notations des massifs roche $L x$, Revue francaise de géotechniqua, $\pi^{\circ} 97$ 2001, p. 53-60.

Mayeu B., Fabre D. - Mesure et mod gation des contraintes naturelles. Application au projet de tunthel ferrovialre
Maurienne-Ambin. Bull. Eng. Geod. \& Erv., vol. 58. 1999, 45-59.

Mayeur B., Fabre D. - Measurement and modeling of in situ rock stresses in the Ambin Massif [Franco-Italiar. Alps). Proc. 37th U.S. Rock Mech. Symp. "Wait Rock 1999". Balkema, 1999, p. 1157-1163

Mayeur B. - Mesure et modelisation des contraintes dars les massifs rochew. Application au tunnel profond Maurienne-Ambin. These de doctorat Geomécanique, Grenoble, 1999, 327 p. [2 vol.].

Muller B. Loback M.L.. Fuchs K. Mas tin L., Gregerser. S., Pavoni N., Stephnasson O., Ljunggren C. - Regiona] patterns of tectonic stress in Europe. J of Geophys. Res., vol. 97. B8. 1992. p. 11783-11803.

Panet M. - La stabitté des ouvrages souterrains. Soutênement et revêtement. LCPC Paris. Papport de recherches $n^{\circ} 28$. $1973,108 \mathrm{p}$

Savage W.Z., Swolfs H.S.. Amadei B. - Od the state of stress in the near surface of the Earth's crust. Pure \& Appl. Geophys. 138, 1992, 0.207-228.

Sokolovski V. - Statios of gramular media. Perganon Press, $2^{m}$ ed, 1965, $220 \mathrm{p}$.

Talobre \$. - La mécanique des roches. Dunod. Paris, 1957

Timoshenko S.P. Goodier J.N. - Theory of elasticity. McGraw-Hil, New York, $3^{\text {put }}$ ed. $1970,567 \mathrm{p}$.

Wheveau Y. Cornet F.H. Destoches J. Blumling P. - Complete in situ stress deternmation in an argilite sedinentary formation. Phys. B Chem, of the Earth. 2007, 19.866-878. 